\title{
PESQUISA QUALITATIVA TRANSVERSAL SOBRE BULLYING NO GRUPO DE PESQUISA EM HUMANIDADES E SOCIEDADE CONTEMPORÂMEA DO IFMT (2016 A 2019)
}

\author{
Raquel Martins Fernandes ${ }^{1}$, Isabel Cristina Silva ${ }^{2}$, Leyze Grecco ${ }^{3}$, Carolina Guimarães \\ Santos ${ }^{4}$, Luiz Carlos de Morais Fernandes ${ }^{5}$ e Silbene Rosa Paoliello ${ }^{6}$ \\ 1Universidade Federal da Paraíba - UFPB. IFMT (PPGEn / IFMT). Brasil, raquel.fernandes@blv.ifmt.edu.br \\ $2,3,6$ \\ Instituto Federald de Mato Grosso-IFMT (PPGEn/IFMT), Brasil, ${ }^{2}$ isabel.silva@plc.ifmt.edu.br, \\ ${ }^{3}$ leyzegrecco@gmail.com, ${ }^{6}$ silbene10@gmail.com \\ 4,5 Universidade Federal de Minas Gerais - UFMG, Brasil, ${ }^{4}$ carolgs124@gmail.com, ${ }^{5}$ luiz.morais@ifmg.edu.br
}

\begin{abstract}
Resumo. O objetivo deste trabalho é analisar as pesquisas realizadas por um Grupo de Pesquisa, entre os anos de 2016 a 2019 e, ainda, apresentar as contribuições dessa pesquisa sobre a violação dos Direitos Humanos e o combate ao bullying. Adotou-se a metodologia descritiva de documentação indireta documental, na qual foram analisados 41 textos científicos entre artigos e trabalhos apresentados em eventos publicados entre 2016-2019. Como resultado destacam-se as palavras-chave: Ensino (23 vezes); Bullying (24 vezes), metodologia qualitativa (25 vezes). Os referenciais teóricos de destaque: Bauman, Abramovay, Oliveira, Foucault, Bauer, Gaskel. Como resultado percebe-se a reflexão sobre a importância de conhecer e confrontar as violações dos direitos humanos no contexto educacional e como estas alcançam status de legitimação e legalização. $\mathrm{E}$, por último, observou-se que há necessidade de implantação de um currículo e políticas públicas que permitam estratégias de enfrentamento a violência.
\end{abstract}

Palavras-chave: Direitos Humanos; Bullying; Ensino.

\section{CROSS-SECTIONAL QUALITATIVE RESEARCH ON BULLYING IN A RESEARCH GROUP IN HUMANITIES AND CONTEMPORARY SOCIETY OF IFMT (2016 A 2019)}

\begin{abstract}
The objective of this work is to analyze the research carried out by a Research Group, between the years 2016 to 2019, and what were the contributions on the violation of Human Rights and the fight against bullying. The descriptive methodology of indirect documentary documentation was adopted. 41 scientific texts were analyzed among articles and works presented in events published between 2016-2019. As a result, the keywords stand out: Teaching (23 times); Bullying (24 times), qualitative methodology (25 times). Theoretical references: Bauman, Abramovay, OLIVEIRA, Foucault, Bauer, Gaskel. As a result, it is clear that the group promoted reflection on the importance of knowing and confronting human rights violations in the educational context and perceiving how they achieve legitimation and legalization status, it was observed that there is a need for a curriculum and policies that allow strategies to confront violence.
\end{abstract}

Keywords: Human Rights; Bullying; Teaching.

\section{INTRODUÇÃO}

O Grupo de Pesquisa em Humanidades e Sociedade Contemporânea do IFMT (GPHSCIFMT) iniciou-se em 2008 com alguns professores de filosofia, com o objetivo de estudar referenciais filosóficos para compreender a sociedade contemporânea. A complexidade da sociedade contemporânea, o processo de formação continuada dos integrantes e as atividades de pesquisa do grupo evocavam um caráter interdisciplinar. Atualmente o Grupo de Pesquisa conta com pesquisadores de várias áreas entre ciências humanas, sociais e até 
mesmo ciências naturais e exatas e busca estudar temas emergentes a partir de diferentes concepções teóricas entre diversos autores, minuciando e analisando de diferentes ângulos os mesmos fenômenos. Em outros termos, o Grupo de Pesquisa, coerente com sua estrutura multidisciplinar, produz diferentes recortes de um mesmo sujeito pesquisado, buscando encontrar, na multiplicidade de experiências e visões teóricas de seus pesquisadores, os conceitos e métodos adequados a uma compreensão interdisciplinar de um mesmo objeto. grupo atua em três diferentes linhas de pesquisa, são elas: Ciência, Trabalho e Tecnologia (oito pesquisadores); Filosofia Política (dois pesquisadores); Vulnerabilidade social, educação e direitos humanos (10 pesquisadores).

Além da pesquisa, o grupo devolve atividades de ensino e extensão, com ações que envolvem a própria comunidade escolar. Atua em processo de verticalização dos níveis de ensino onde pesquisadores se envolvem com estudantes desde o ensino médio passando pelo ensino superior até os níveis stricto senso, neste processo alunos doutorandos auxiliam os mestrandos, que auxiliam os graduandos e os alunos de ensino médio; estes últimos trazem no teor de suas ações o protagonismo jovem como referência de conteúdo e método no contexto pós-moderno.

O grupo se propõe a formar pesquisadores preocupados não apenas com o objeto de pesquisa, mas com a problemática real em que se insere este objeto. Os dados diagnósticos apresentam possíveis soluções que cooperam com a propositura de políticas educacionais. Um exemplo é a BNCC (Base Nacional Comum Curricular da Educação Básica/ homologada 14/12/2018) cujo objetivo é a formação humana integral dos educandos para a construção de uma sociedade mais justa, democrática e inclusiva.
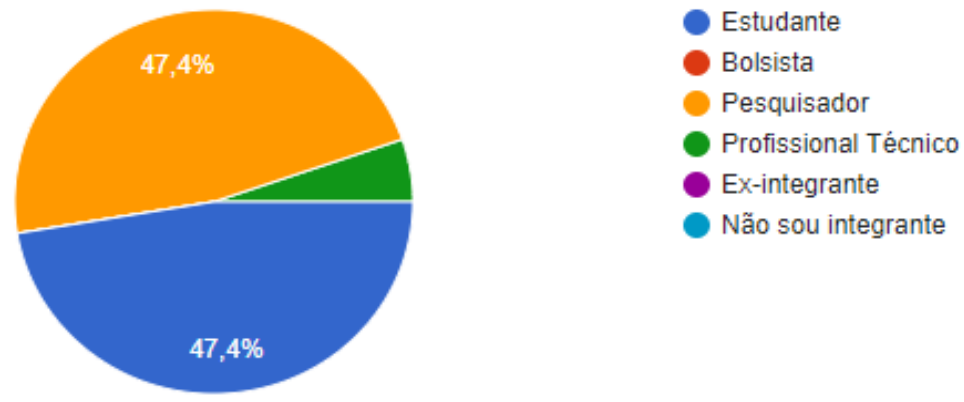

Figura 1. Integrantes do GPHSC-IFMT.

Fonte: autor próprio, 2019. 
A figura1 representa os integrantes do GPHSC, sendo $47,4 \%$ estudantes do ensino médio integrado, graduandos e mestrandos e 47,4\% professores pesquisadores nas áreas de Linguagem, Ciências Humanas e Sociais, Matemática, Ciências da natureza e Tecnologia. O resultado desta parceria pode ser visto na qualidade da pesquisa, diversidade dos temas e artigos publicados pelo Grupo.

Em 2017, um artigo sobre os fundamentos da pesquisa qualitativa a partir das experiências dos pesquisadores, mostra resultados sobre o falso dualismo entre os termos qualitativo e quantitativo na pesquisa em educação. A partir de reflexões filosóficas, concebendo que a quantidade evoca a qualidade do objeto, tal qual a qualidade é um aspecto da quantidade; os pesquisadores apontam um falso dualismo e apresenta a vertente da concepção do elemento qualitativo, como o recorte subjetivo que o pesquisador faz da realidade observada (Mota et al., 2017). Diante da concepção deo dualismo o grupo de pesquisa procura contribuir para compreender a realidade social em múltiplos olhares, na contribuição de várias áreas em torno da busca de solução de problemas sociais.

Em 2016, teve início à pesquisa sobre a "Violação dos Direitos Humanos e Bullying no contexto escolar": diagnóstico e proposta de intervenção com base no empoderamento dos alunos aprovada pelo Comitê de Ética em Pesquisa e esteve em andamento durante 2016, 2017, 2018, 2019 e prossegue em 2020. O GPHSC-IFMT propõe e desenvolve uma análise de natureza qualitativa (Mota et al., 2017), utilizando-se como método inicial de produção de dados um questionário com perguntas abertas e de múltipla escolha, aplicado aos alunos do ensino médio, ensino superior, e, mais recentemente, ensino fundamental; após o diagnóstico, a pesquisa em uma segunda etapa, envolve entrevistas, grupos focais, estudos de caso, rodas de conversa e proposituras de eventos, conforme o foco de cada temática, pesquisador ou realidade diagnosticada.

A perspectiva de investigação dos dados visa compreender e interpretar determinados comportamentos, opiniões e as expectativas dos indivíduos dentro do contexto escolar. Entender o sujeito, moldado pela globalização e envoltos numa complexa teia de informações em tempo real, assimilar determinados comportamentos e questionar o óbvio, são procedimentos importantes para indicar as melhores ações no sentido de provocar a reflexão e a construção do conhecimento em um método qualitativo. Segundo Velho (1997, p. 131), "o processo de estranhar o familiar torna-se possível quando somos capazes de confrontar intelectualmente, e mesmo emocionalmente, diferentes versões e interpretações existentes a 
respeito de fatos". Tal apontamento vem corroborar com as diferentes visões de um mesmo objeto, principalmente na compreensão transversal que se propõe o grupo sob os diferentes olhares dos pesquisadores, estudantes e técnicos.

Os dados aqui analisados dizem respeito aos resultados apresentados pelo grupo à comunidade científica, como resultante da junção do trabalho de vários pesquisadores, de diversas áreas com o objetivo de construir um diagnóstico de compreensão e prevenção do bullying nos mais variados contextos educacionais. A produção de dados foi realizada por um grupo de pesquisadores para cada instituição, já a tabulação e análise foi realizada por outro grupo, o que possibilitou compreender o bullying por diversos ângulos e perspectivas.

A pesquisa em 2016 e 2017 foi realizada em 7 (sete) unidades de ensino, sendo 4 (quatro) Institutos Federais de Mato Grosso (IFMT), 2 (duas) escolas estaduais de Cuiabá e 1 (uma) escola privada (Cuiabá). Em 2019, a pesquisa contou com a adesão de novas escolas e pesquisadores, num total de 25 escolas cadastradas e 11 escolas que efetivamente participaram da aplicação do questionário.

A pesquisa abarca uma abordagem qualitativa que, para Bauer e Gaskell (2017), possui em sua característica a busca pelos significados. As técnicas de produção de dados utilizadas nas difererentes etapas de pesquisa constituíram-se de observação, aplicação de um questionário com questões de múltipla escolha e de perguntas com respostas abertas, livres, além de uma entrevista estruturada. O método foi definido através de debates em reuniões periódicas. O questionário é composto por treze questões, assim dividido: questões de 1 (um) a 8(oito) referem-se aos dados do aluno sujeitos da pesquisa, questão 9 (nove) subdivida em 22 (vinte e dois) itens, referem-se especificamente ao tipo de bullying. Questões de 10(dez) a 12(doze), referente ao perfil dos sujeitos envolvidos no processo (agressor, espectador, forma, aspectos culturais, dentre outros) e a questão 13, tem como referência sugestões para o combate ao bullying. Em 2019, o questionário ficou composto com perguntas abertas e fechadas, sendo 03 interpretativas e 24 questões fechadas (objetivas). O questionário é disponibilizado online, através de formulário do google-drive. Questões de 10 (dez) a 12 (doze), referente ao perfil dos sujeitos envolvidos no processo (agressor, espectador, forma, aspectos culturais, dentre outros) e a questão 13, tem como referência sugestões para o combate ao bullying que nortearam o recolhimento das informações de forma anônima. Fazem agressões virtuais? Humilham-me por minha orientação sexual? Fazem piada por minha cor e raça? Colocam-me apelidos vergonhosos? Foram algumas das questões 
elencadas no questionário online que forneceram dados para um diagnóstico dos casos de bullying e violação dos direitos humanos nas instituições pesquisadas.

Após aplicação das técnicas de coleta de dados, eles foram organizados, sistematizados e analisados pelos componentes do GPHSC-IFMT; a análise diagnóstica, o panorama geral levou os pesquisadores a focar e aprofundar em temáticas específicas com o retorno a campo, com novos instrumentos de coleta de dados, tais como entrevistas, rodas de conversas, entrevistas em grupos, projetos de intervenção, dentre outras. As temáticas específicas pesquisadas pelo grupo são: gênero, racismo, bullying e questões sociais, educação em direitos humanos, corporeidade, sexualidade, inclusão e exclusão escolar, identidade, homofobia, currículo escolar, políticas públicas, segregação de etnias indígenas, mídias sociais, cyberbullying, violência escolar, violência e desenvolvimento cognitivo, ensino de ciências, educação matemática, panorama da sociedade contemporânea e outros.

Nesse panorama da sociedade contemporânea e com a diversidade de temas, em 2016 e 2017 foram publicados 15 trabalhos - Seminário Educação 2016, IE/UFMT, Cuiabá/MT (01 trabalho completo); VI SECAMPO 2016, Mamaguapé/PB (01 trabalho completo); IX Seminário Internacional - As Redes Educativas e as Tecnologias 2017, Rio de Janeiro: UERJ (03 trabalhos completos); Congresso Ibero-Americano em Avaliação Qualitativa (CIAIQ) Salamanca/Espanha, 2017 (03 trabalhos completos, sendo um artigo publicado em revista científica); $69^{\circ}$ Reunião anual da SBPC 2017, Belo Horizonte UFMG (2 resumos expandidos); Seminário de Educação 2017, UFMT, Cuiabá (03 trabalhos completos); IV Congresso Nacional de Educação 2017, João Pessoa (03 trabalhos completos). Em 2018, o grupo de pesquisa participou de cinco eventos científicos no Brasil (WORKIF 3, JENPEX Cuiabá Bela Vista 8, SEMIEDU 3, CONEDU 4, JEPEX Pontes e Lacerda 1), perfazendo um total de 19 trabalhos apresentados, dentre eles oito resumos simples, dois resumos expandidos e nove trabalhos completos. Em 2019 foram publicados 28 trabalhos: Revista Kroton (01 artigo); Revista Eletrônica Acervo Saúde / Electronic Journal Collection Health (01 artigo); Revista Impulso, Piracicaba (01 artigo); III Conepi UFPB / Joaõ Pessoa-PB (03 trabalhos completos); II SEI (09 resumos simples); VI Jenpex Cultural - IFMT / Cuiabá / campus Bela Vista (03 resumos expandidos); Jenpex / Cuiabá / campus Octayde (06 resumos simples); VIII Simpósio Nacional de Ciência, Tecnologia e Sociedade (01 trabalho completo); Capítulos de Livro (03) e a produção de uma edição especial em E-BOOk, contendo doze artigos a ser publicada em 2020. 


\section{METODOLOGIA}

A metodologia utilizada é descritiva de documentação indireta documental. Este artigo propõese a descrever como os trabalhos foram apresentados à comunidade científica e qual a contribuição real que o GPHSC-IFMT traz à formação de pesquisadores, ao trabalho multi e transdisciplinar, bem como a qualificação de elementos que permitam repensar as ações da sociedade civil frente ao problema do bullying e a violação dos direitos humanos. Analisou-se as publicações do grupo, adotou-se a pesquisa qualitativa de cunho transversal, que diz respeito à possibilidade de se instituir, a partir de múltiplos olhares (áreas, aportes teóricos, instrumentos de pesquisa diversificados, equipe multiprofissional) um resultado qualitativo e dinâmico sobre o tema em questão.

Optou-se por uma pesquisa de cunho documental, uma vez que foram analisadas as produções científicas do grupo.

Analisou-se os artigos publicados pelo grupo no período de 2016-2018, totalizando 34 artigos. Em 2016 e 2017 foram publicados 15 trabalhos; em 2018, 19 trabalhos. Dos 28 trabalhos apresentados em 2019 foram analisados 07 artigos e/ou trabalhos completos. Ao todo foram selecionados 41 trabalhos científicos.

Das produções dos primeiros anos foram incluídas todas, já do ano de 2019 foram analisados somente os trabalhos completos, o critério foi utilizado devido ao número expressivo de publicações.

Uma vez selecionados os textos, foram retirados dos mesmos alguns elementos chave que permitem ter uma compreensão geral da produção do grupo (palavras-chave, metodologia e aporte teórico).

Após a tabulação destes dados e observação das ocorrências, os artigos foram novamente analisados, para permitir a construção de um panorama do perfil das produções do GPHSCIFMT

Após a leitura foram construídas as seguintes tabelas com os resultados: 
Tabela 1 - Palavras-chave dos trabalhos científicos 2016-2019 - GPHSC-IFMT

\begin{tabular}{|c|c|c|c|}
\hline Palavras-chave & $\begin{array}{l}\mathrm{N}^{\circ} \mathrm{de} \\
\text { vezes }\end{array}$ & Palavras-chave & $\begin{array}{l}\mathrm{N}^{0} \mathrm{de} \\
\text { vezes }\end{array}$ \\
\hline $\begin{array}{l}\text { Análise de Discurso Crítica } \\
(A D C) \text {, }\end{array}$ & 01 & Formação & 01 \\
\hline Agropecuário & 01 & Interdisciplinaridade Cultural & 01 \\
\hline Bullying & 24 & Gestão da Qualidade & 01 \\
\hline Chiquitanos & 01 & Gênero & 01 \\
\hline Cotidiano escolar & 01 & IFPB & 01 \\
\hline Cotidianidades & 01 & IFMT & 01 \\
\hline Ciências Humanas & 02 & Institutos Federais & 01 \\
\hline Currículo escolar & 02 & Identidade & 03 \\
\hline Cyberbullying; & 02 & Inclusão & 02 \\
\hline CURTABLV & 01 & Intimidação Sistemática & 02 \\
\hline Cibercultura & 01 & Normatização & 02 \\
\hline Direitos Humanos & 13 & Novas tecnologias educacionais & 01 \\
\hline Diferença & 01 & PDI. & 04 \\
\hline Desenvolvimento Regional & 01 & Pesquisa & 05 \\
\hline Discursos & 02 & Pesquisa Qualitativa & 02 \\
\hline Educação & 06 & Reconhecimento & 01 \\
\hline Escola Pública & 02 & Racismo & 01 \\
\hline Extensão & 01 & Redes sociais & 01 \\
\hline Economia Criativa & 01 & Sistemática & 01 \\
\hline Ensino & 23 & Sustentabilidade & 01 \\
\hline Ensino Médio & 01 & Sistemática & 01 \\
\hline Exclusão & 02 & Sustentabilidade & 01 \\
\hline Educação inclusiva & 01 & Teorias da Educação & 01 \\
\hline Estudantes & 01 & Violência & 05 \\
\hline Ensino-aprendizagem & 01 & Vulnerabilidade Social & 01 \\
\hline Educação ambiental & 01 & Violação & 01 \\
\hline Ética ambiental. & 01 & Vivências & 01 \\
\hline Formação humana & 01 & Violência Escolar & 06 \\
\hline Fenomenologia & 02 & & \\
\hline
\end{tabular}

Fonte: autor próprio, 2019

A Tabela 1 representa o número de vezes que a palavra aparece entre as palavras-chave dos artigos; publicados de 2016-2019.

Destacam-se as palavras-chave: Ensino (23); Bullying (24); Direitos Humanos (13); Violência (8); Educação (6); Pesquisa (5); Identidade (3); Inclusão (2); Plano de Desenvolvimento Institucional - PDI (4). As palavras-chave traduzem o sentido das pesquisas na busca de estudo e resolução de conflitos e os aspectos que permeiam a discussão, com foco no ensino e educar para a paz e cidadania como tarefa necessária, frente à realidade social de nossos dias. 
Tabela 2 - Metodologia dos trabalhos científicos 2016-2019 - GPHSC-IFMT

\begin{tabular}{l|c}
\multicolumn{1}{c|}{ Metodologia } & $\mathbf{N}^{\circ}$ de vezes \\
\hline Qualitativa & 15 \\
Qualitativa - Descritiva e Interpretativa & 10 \\
Pesquisa Bibliográfica e documental & 08 \\
Historiográfico & 02 \\
Análise de Discurso & 02 \\
Fenomenologia & 02 \\
Quali-quanti & 01 \\
Documentação direta & 01 \\
\hline
\end{tabular}

Fonte: autor próprio, 2019

A Tabela 2 apresenta as metodologias caracterizadas pelo cunho qualitativo, dentre elas destacam-se: Qualitativa (15); Qualitativa- Descritiva e interpretativa (10); Pesquisa bibliográfica, documental e explicativa (08); Método Historiográfico (02), Análise de Discurso (02), Fenomenologia (02); Quali-quanti (01) e Documentação direta (01). De um modo geral, percebe-se que a dinâmica do grupo em abordagem geral qualitativa, não é ater a um só método de procedimentos, mas utilizar metodologias que se complementam para compreender o fenômeno pesquisado, este é o aspecto estudado em reuniões do grupo, como elemento de transversalidade, o uso de técnicas, procedimentos e métodos diversificados.

A transdisciplinaridade incorpora a integração do conhecimento científico a outros modos de produção de conhecimento construídos historicamente pela humanidade, envolvendo várias áreas do saber e diferentes referenciais das ciências humanas e sociais, parte do pressuposto epistemológico de que a realidade social é constituída por diferentes níveis em coexistência (Mota et al., 2017).

Tabela 3 - Referencias Teóricos dos trabalhos científicos 2016-2019 - GPHSC-IFMT

\begin{tabular}{l|c}
\hline \multicolumn{1}{c|}{ Referenciais Teóricos } & $\mathbf{N}^{\mathbf{0}}$ de vezes \\
\hline Michel Bauman & 02 \\
Miriam Abramovay e Mary Garcia & 03 \\
Castro & 02 \\
Ivone Martins de Oliveira & 02 \\
Foucault & 04 \\
Martin W Martins \& George Gaskel. & 02 \\
César Coll (2000, 1998, 1996) & 02 \\
Marin e Pimenta (2018) & 02 \\
Silva (2015) e Morin (2000). & 02 \\
Zabala (2002, 1998)
\end{tabular}

Fonte: autor próprio, 2019 
Os referencias em destaque foram: Zygmunt Bauman; Miriam Abramovay e Mary Garcia Castro; Ivone Martins de Oliveira, Michel Foucault; Martin W. Bauer e George Gaskel. Dentre vários outros: Dilthey, Weber, Merleau-Ponty, Hall, e Barth (2011); Almeida, Lisboa, e Caurcel (2007); Olweus (1995); Calhau (2009); Chalita (2008); Fante (2004, 2005); Mota (2016); Zanella, e Trevisol (2014); Louro (1997); Cerqueira (2016); Guimarães (2009); Issler, e Carvalho (2010); Pereira (2011); Santos (2014); Chauí (2017); Carvalho (2018); Certeau (2004); Beaudoin (2006); Canário (2006); Chalita (2008); Mota (2017); Canclini (2010); Beaudooin (2006).

Os autores citados possuem perspectivas teórias específicas dentro do campo de atuação dos mesmos. Permitem entender a transformação nas relações humanas de uma forma "liquida", mudanças adaptáveis e moldáveis (Bauman, 2005). Interpretando a escola, como uma instituição que possui uma missão ideológica de adequar os sujeitos à vida em sociedade, produzindo discursos e moldando comportamentos, que carecem de uma análise crítica de suas estruturas (Foucault, 2004). Elementos da realidade contemporânea que precisam ser questionados, visando a formação humanística e o combate às vulnerabilidades sociais e violência escolar.

O GPHSC-IFMT, que conta com pesquisadores das várias áreas das ciências humanas e sociais, busca interpretar a sociedade contemporânea a partir de diferentes concepções teóricas e diferentes autores e, também, descreve e analisa de diferentes ângulos os mesmos fenômenos. O grupo é caracterizado pela plasticidade conceitual que viabiliza a flexibilidade, maleabilidade e elasticidade que encontra na diversidade de experiências e visões teóricas de seus pesquisadores, os conceitos e métodos adequados a uma compreensão interdisciplinar do sujeito pesquisado. A pesquisa qualitativa transversal assume assim um caráter multidisciplinar porque várias pessoas, pesquisadores, profissionais de diferentes áreas emitem seus pontos de vista acerca de um único objeto. Multidisciplinaridade como o exame, a avaliação e definição do mesmo objeto sob diversos olhares de diferentes princípios, conceitos e vertentes. Prevalecendo, inclusive, a pluralidade de opiniões e o respeito aos diferentes posicionamentos políticos que podem decorrer dos resultados alcançados.

\section{CONCLUSÕES}

A pesquisa referente ao bullying tem sua relevância social tendo em vista os malefícios que este traz para a vida dos envolvidos. É importante formar cidadãos que respeitem as diferenças, com consciência de seus direitos e deveres. Na contemporaneidade, houve 
grandes mudanças nos cenários político e econômico no país, e, ainda, um novo olhar para a ciência, a arte; a sociedade também teve suas transformações e junta-se a estas mudanças, os problemas que delas decorrem. O bullying é o reflexo da violência social e um fenômeno complexo que merece, portanto, ser abordado cientificamente.

Após análise dos artigos publicados entre 2016-2019, percebe-se que foi realizada a qualificação de elementos que permitem repensar as ações da sociedade civil frente ao problema do bullying e a violação dos direitos humanos e contribui com as discussões na política educacional. Os resultados da pesquisa do grupo até o momento permitiram fomentar discussões sobre os temas abordados, entre os próprios pesquisadores, as instituições pesquisadas e a comunidade escolar; mostrando a possibilidade de construção de referenciais e atividades interdisciplinares, multidisciplinares e transversais que propiciem um melhor entendimento do que significa os Direitos Humanos e a importância dos mesmos para a construção de uma sociedade mais justa e igualitária onde prevaleça a convivência saudável entre os indivíduos.

\section{REFERÊNCIAS}

Abramovay, M. (2003). Violências nas escolas et alii. Brasília: UNESCO Brasil, REDEPITÁGORAS, Coordenação DST/AIDS do Ministério da Saúde, a Secretaria de Estado dos Direitos Humanos do Ministério da Justiça, CNPq, Instituto Ayrton Senna, UNAIDS, Banco Mundial, USAID, Fundação Ford, CONSED, UNDIME.

Bauman, Z. (2005). Identidade. Rio de Janeiro: Zahar.

Bauer, M. W., \& Gaskell, G. (2017). Pesquisa Qualitativa com Texto Imagem e Som. Tradução de Pedrinho. Guareschi Petrópolis. RJ: Vozes.

BNCC-EM, Resolução CNE/CEB no 3/2018. Homologada em 17/12/2018. Disponível: http://portal.mec.gov.br/docman/dezembro-2018-pdf/104101-rcp004-18/file.

Castro, M. G., \& Abramovay, M. (2006). Relações raciais na escola: reprodução de desigualdades em nome da igualdade. Brasília: UNESCO, INEP, Observatório de Violências nas Escolas.

Foucault, M. (2004). Tecnologias de Si. Verve, 6. São Paulo: PUC.

Foucault, M. (1997). História da sexualidade. In Microfísica do poder (pp. 243-270). Rio de Janeiro: Graal.

Mota et al. (2017). Pesquisa qualitativa em Educação. Indagatio Didática, 9(3), 79-98. http://revistas.ua.pt/index.php/ID/article/view/6056.

Mota, R.M.F, Silva, I.C., Santos, G.S., \& Souza, J.A. (2017). Estudo sobre a violação dos direitos humanos e Bullying no IFMT. In Seminário de Educação 2017. Brasil, MT, Cuiabá: UFMT.

Mota, R. M.F., Oliveira, P. A., Colpani, D., \& Oliveira, R. R. (2018). Uso das redes sociais na educação profissional e tecnológica. Impulso, Piracicaba, 28(73), 35-52.

Silva, V. C. G., Mota, R. M. F., \& Campos S. M. G. (2019). Bullying e Processos de Escolarização: Diálogos e Perspectivas Pedagógicas Bullying and the Schooling Processes: Dialogues and Pedagogical Perspetices. Rev. Ens. Educ. Cienc. Human. 
Morin, E. (2000). Ciência com consciência. Rio de Janeiro: Bertrand.

Oliveira, A. M., Queiroz, F.C., \& Mota, R. M. F. (2019). Bullying no contexto escolar: apontamentos à luz de Foucault. In: Anais, IX Seminário Internacional - As Redes Educativas e as Tecnologias, Rio de Janeiro: UERJ. Recuperado de http://www.seminarioredes.com.br/ixredes/adm/trabalhos/diagramados/TR563.pdf.

Oliveira, C., Souza, V., Oliveira, F., \& Silva, V. C. G. (2017). Violência escolar no Brasil: desafios em curso na educação do século XXI. In: Anais, Congresso Ibero-Americano em Avaliação Qualitativa, Salamanca: CIAIQ. Recuperado de http://proceedings.ciaiq.org/index.php/ciaiq2017/article/view/1540/1497.

Oliveira, P.A., Duarte, V.C.O., \& Araújo, M.G.O. (2017). Educação, gênero e formação humana: o processo de ensino aprendizagem agropecuário e a ressignificação da mulher no mercado de trabalho. In: Anais, IX Seminário Internacional - As Redes Educativas e as tecnologias, Rio de Janeiro: UERJ. Recuperado de http://www.seminarioredes.com.br/ixredes/adm/trabalhos/diagramados/TR547.pdf.

Oliveira, P.A., Mota, R.M.F., Oliveira, V.C.D., \& Silva, V.C.G. (2017) O Bullying e o ensino aprendizagem na vivência e cotidianidade das mulheres na educação técnica em agropecuária. In: Seminário de Educação 2017. Brasil, MT, Cuiabá: UFMT.

Oliveira, P.A., Mota.R.M.F. (2016). Educação, diversidade de gênero e inclusão: o direito de uso do nome social pelas travestis e transexuais nas instituições de ensino do Brasil. In: VI SECAMPO, Brasil, PB, Mamanguapé.

Oliveira, P.A., Silva, C.G.S., Mota, R.M.F., Duarte, V. C.O., \& Assumpção, Y.O. (2017). Violação dos direitos humanos e bullying: a sociabilidade no cotidiano escolar. In: $69^{a}$ Reunião anual da Sociedade Brasileira para o Progresso da Ciência (SBPC) 2017, Brasil, MG, Belo Horizonte: UFMG.

Oliveira, P.A., Mota, R.M.F., \& Alves, W.L.C. (2017) O Bullying e o ensino-aprendizagem agropecuário: vivências e cotidianidades. In: IV Congresso Nacional de Educação, João Pessoa: Realize.

Silva, V. C.G., Souza, J. A., Oliveira, C.E., \& Mota, R. M.F. (2017). Cotidiano escolar do IFMT: análise do discurso de proposituras de combate ao bullying. In: Seminário de Educação 2017, Brasil, MT, Cuiabá: UFMT.

Silva, V.C.G., Assumpção, Y.O., Neiva, M.A.B., \& Mota, R.M.F. (2016). Violação dos direitos humanos e bullying no contexto escolar: uma realidade que precisar ser enfrentada. In: Anais, Seminário de Educação 2016, Brasil, MT, Cuiabá: UFMT. Recuperado de http://srvdotnet.com.br/semiedu2016/FrmAnais.aspx?eventoUID=117.

Silva, V.C.G., Souza, J.A., \& Neiva, M.A.B. (2017). Bullying e a construção da identidade do adolescente. In: Anais, IX Seminário Internacional - As Redes Educativas e as Tecnologias 2017, Rio de Janeiro. Recuperado de http://www.seminarioredes.com.br/ixredes/adm/trabalhos/diagramados/TR564.pdf, Rio de Janeiro: UERJ.

Souza, J.A., Mota, R.M.F., Silva, V. C.G., \& Anjos, D.F. (2017). Bullying, identidade e direitos humanos no contexto escolar. In: IV Congresso Nacional de Educação, João Pessoa: Realize.

Velho, G. (1997). As Ciências Sociais Nos Últimos 20 Anos: Três Perspectivas. In: Rev. bras. Ci. Soc.[online]. ISSN 0102-6909. http://dx. doi. org/10.1590/S0102. 